\title{
Design and Construction of a Device for Obtaining Three-Dimensional Coordinates in Different Topographic Surfaces Through the Use of Wireless Networks, Gps and Altimeter
}

Marlene Ballestas; Vladimir Pinzón; Ruben Guerra; Alonso Barrera; Jesús Vergara

\begin{abstract}
The present investigation is done through processes that permit to find the value of the unknown term " $\mathrm{R}$ ". The procedure for data collection disposed the transmitter and the receptor two meters apart, measuring the value of the parameter RSSI a total of twenty times; repeating this process every two meters, until the forty meters. The procedure for data collection disposed the transmitter and the receptor two meters apart, measuring the value of the parameter RSSI a total of twenty times; repeating this process every two meters, until the forty meters. In relation to the statistical treatment of the data, there were applied dispersion measurements or variability of the sample, such as the sample's variation, the standard deviation of the sample and the error. The results established the existence of the relation between the two principal variables involved in the study. Potency $(\mathrm{dBm})$ as independent variable and the distance as dependent variable, in conclusion, this is prove that the bigger the distance between the transmitter and the receptor is, the less power it expresses.
\end{abstract}

\section{Keywords}

Three-dimensional; Topography: Planimetry 